\title{
Development of gas wire chambers for in-beam charged particle detector in the KОTO experiment
}

\author{
Kota NAKAGIRI*, for the KOTO Collaboration \\ Kyoto University \\ E-mail: n-kotadscphys.kyoto-u.ac.ip
}

Low-mass thin gap chambers were developed as an in-beam charged-particle detector for the KOTO experiment. Three chambers are placed in line along the beam axis. The detection efficiency of $99.6 \%$ was achieved in each module and $99.9 \%$ was achieved as the whole detector system. This detector is used as a veto counter and operated in high flux of gammas and neutrons. Plastic scintillator strips with 3-mm thickness were used in the previous run and caused significant signal loss due to accidental hits by beam-contained gammas and neutrons. Using low-mass thin gap chamber, the interaction rate with gammas and neutrons decreases and the signal loss becomes small. With the rate reduction of $1 / 3$, although its 30 -ns veto time window is twice longer than old one, the signal loss caused by upgraded BHCV is expected to be reduced to $2 / 3$ that caused by old BHCV.

Flavor Physics \& CP Violation 2015

May 25-29, 2015

Nagoya, Japan

\footnotetext{
* Speaker.
} 


\section{1. $K_{L} \rightarrow \pi^{0} v \bar{v}$ decay and the KOTO experimant}

The $K_{L} \rightarrow \pi^{0} v \bar{v}$ decay is a CP-violating rare decay. This decay is a flavor changing neutral current decay and caused via loop diagram (Figure.W).

If new processes are involved in the decay, the branching ratio would be larger than expected in the

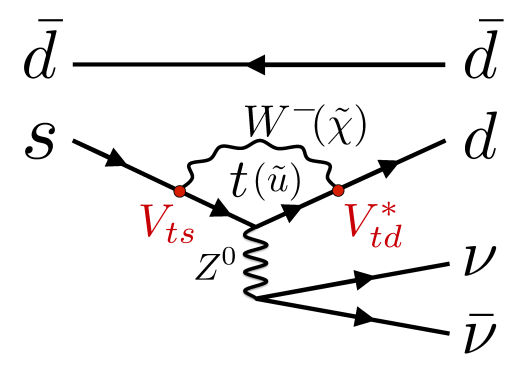

Figure 1: One of the Feynman diagrams of $K_{L} \rightarrow \pi^{0} v \bar{v}$ decay.

Standard Model $\left((2.43 \pm 0.06) \times 10^{-11}[\mathrm{U}]\right)$. This suppressed branching ratio and small theoretical uncertainty $(\sim 2 \%)$ allow us to observe effects from new physics.

The KOTO experiment[[]] aims to study $K_{L} \rightarrow \pi^{0} v \bar{v}$ at Japan Proton Accelerator Research Complex (J-PARC). The first goal of KOTO is to observe $K_{L} \rightarrow \pi^{0} v \bar{v}$ events. KOTO conducted its first physics run in May 2013 and achieved almost the same single event sensitivity as the E391a experiment[[]] which gives the world record of the upper limit of the $K_{L} \rightarrow \pi^{0} v \bar{v}$ branching ratio.

\section{Experimental method of KOTO and Beam Hole Charged Veto (BHCV)}

In the KOTO experiment, candidates of $K_{L} \rightarrow \pi^{0} v \bar{v}$ signal are identified by "two photons and nothing else." The KOTO detector surrounds hermetically decay volume (Figure $\square$ ). A narrowly collimated $K_{L}$ beam generated by bombarding primary $30-\mathrm{GeV}$ protons at the production target is transported to the detector area located 20 meter from the target. We measure two photons from a $\pi^{0}$ decay with an electromagnetic calorimeter ("CsI" in Figure $\mathbb{2}$ ) and ensure there are no other observable particles with hermetic veto counters.

The Beam Hole Charged Veto (BHCV) detector is one of the veto counters and is located at a downstream in-beam area. In order to suppress backgrounds, $99.5 \%$ or higher efficiency to a charged particle is required. BHCV should be insensitive to neutral particles because the $K_{L}$ beam contains a large amount of photons and neutrons ${ }^{1}$. In the previous run performed in May 2013, pairs of a plastic scintillator strip with 3-mm thickness and a PhotoMultiplier Tube (PMT) were used as BHCV. The counting rate of BHCV was $8.4 \mathrm{MHz}$ with 24-kW beam and the gain of the PMTs was unstable due to the high counting rate. Such a high counting rate caused a significant signal loss because any events with a visible energy in a veto counter were rejected. The signal loss is expressed by the function of $R T$, where $R$ is the counting rate and $T$ is the veto timing window of $\mathrm{BHCV}$. The veto timing window is determined by the width of the flight time distribution and time

\footnotetext{
${ }^{1}$ The flux of photons (neutrons) reaches $0.6 \mathrm{GHz}(0.6 \mathrm{GHz})$ with a $300-\mathrm{kW}$ primary proton beam, which is KOTO's designed value of beam intensity.
} 
jitter of BHCV itself. BHCV induced 12\% signal loss with a 15-ns veto window in the previous run (preliminary).

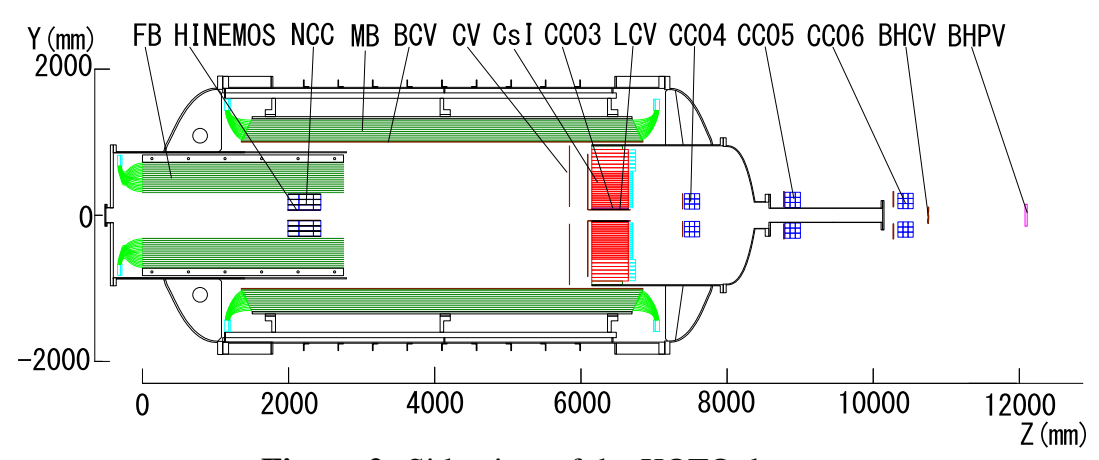

Figure 2: Side view of the KOTO detector.

\section{Upgrade of BHCV}

In order to cope with the gain instability and the signal loss, BHCV upgrade was required. We decided to use wire chambers as BHCV because they contain small amount of material and an interaction rate with beam-contained photons and neutrons is expected to decrease. We adopted a "thin gap chamber" which has a thin wire-cathode gap and is known to be tolerant to high rate condition[回] because $4.1-\mathrm{MHz}$ counting rate is expected under $300-\mathrm{kW}$ proton beam operation. Figure 3$]$ shows the structure of the chamber. Gold-plated tungsten wires $50-\mu \mathrm{m}$ in diameter are

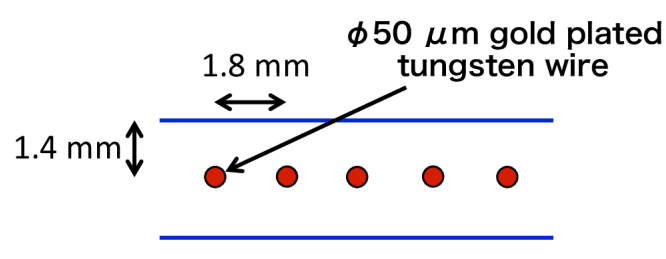

(a)

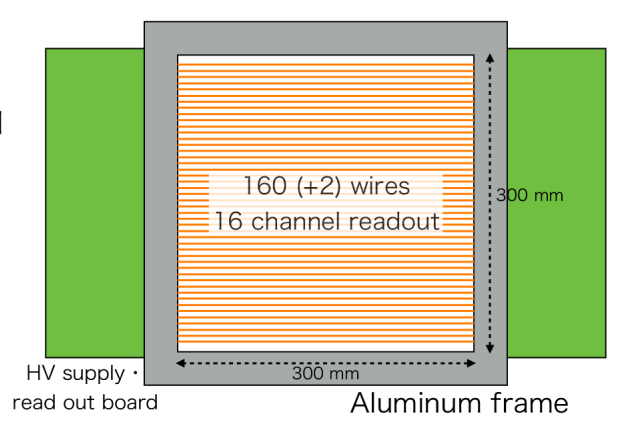

(b)

Figure 3: Cell structure (a) and overview (b) of the chamber.

used as anode wires. It has a 1.8-mm wire spacing and a 1.4-mm wire-cathode gap. It covers an approximately $30 \mathrm{~cm} \times 30 \mathrm{~cm}$ region and 160 sense wires are strung in a module. Ten wires are ganged and read out as a channel. In order to reduce amount of material, we chose a thin graphite coated film with $50-\mu \mathrm{m}$ thickness as a cathode plane. 
The chamber is filled with a 55:45 mixed gas of Tetrafluoromethane $\left(\mathrm{CF}_{4}\right)$ and n-Pentane. Wire chamber has a relatively large time jitter because of the drift time of electron from an ionized point to an amplified region. We chose $\mathrm{CF}_{4}$ gas which is known as a "fast gas" in which electron drift velocity is fast. In order to stabilize the operation, n-Pentane, which is a strong quencher, is mixed.

We produced three chambers and installed them in line along the beam axis. When we require more than two out of three chambers' hits as a particle detection (2-out-of-3 logic), the total inefficiency ( $=1-$ efficiency) of BHCV, $\eta_{\text {tot }}$, becomes lower than that of each module, $\eta$, as follows:

$$
\begin{aligned}
\eta_{\mathrm{tot}} & =\eta^{3}+3 \eta^{2}(1-\eta) \\
& =3 \eta^{2}-2 \eta^{3}<\eta
\end{aligned}
$$

\section{Performance of new BHCV}

We performed a special run at the KOTO beamline for new BHCV efficiency measurement, where upstream beam plug was closed in order to select high momentum muons or pions in the beam. Figure $\$$ shows the measurement setup. Data acquisition trigger was generated by the coincidence hits in old BHCV and an upstream trigger scintillator, which was installed only in this special run. BHPV shown in Figure $\mathbb{\Psi}$ is a photon counter made of aerogel and lead. Its total radiation length is approximately $6.2 \mathrm{X}_{0}$. We also required a hit in the downstream trigger scintillator behind BHPV in the analysis in order to further select penetrating high momentum muons or pions. Figure 1 shows energy deposit distribution of the chamber after the selection. The detection efficiency of $99.6 \%$ was achieved with the threshold at 1/4 Minimum Ionizing Particle (MIP) peak. Figure 6 shows the time jitter of BHCV. Considered with the width of time of flight distribution, which is approximately $10 \mathrm{~ns}$, veto window should be set approximately $30 \mathrm{~ns}$. It is 2 times wider than old BHCV. The counting rate of new BHCV was $1.4 \mathrm{MHz}$ in the run performed in April 2015 and it was 1/3 of that of old one. The signal loss reduction rate by this upgrade is estimated to be $2 / 3$.

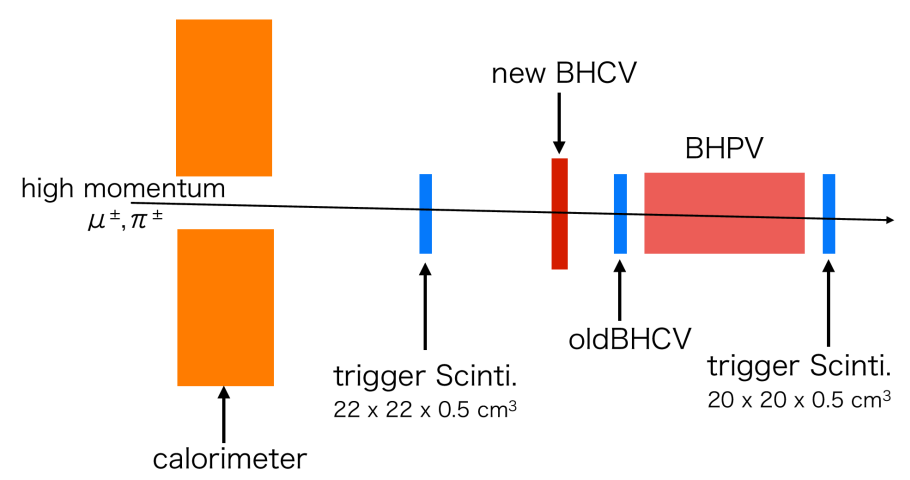

Figure 4: Experimental setup. 


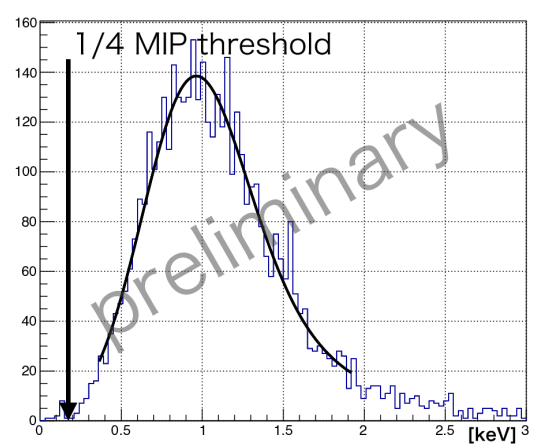

Figure 5: Energy distribution of the chamber.

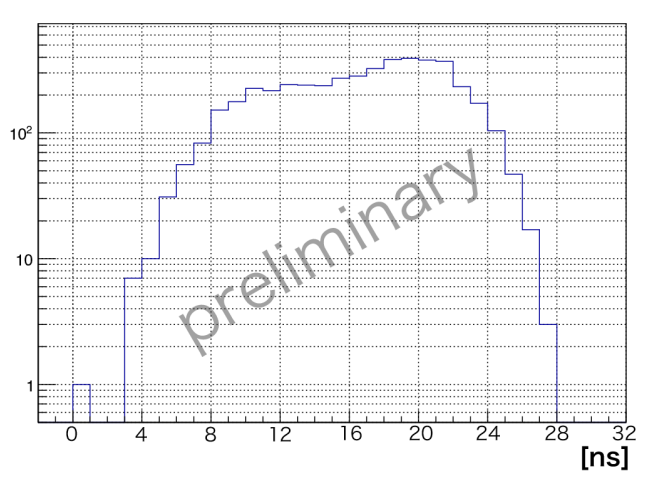

Figure 6: Detection time distribution of BHCV measured from trigger timing.

\section{Conclusion}

We developed low-mass thin gap chambers for an in-beam charged particle detector used in the KOTO experiment. The efficiency of $99.6 \%$ was achieved in each chamber and $99.6 \%$ was achieved as the whole detector system, or 2-out-of-3 logic mentioned in section 3. With the rate reduction of $1 / 3$, although its 30 -ns veto time window is twice longer than old one, the signal loss caused by upgraded BHCV is expected to be reduced to $2 / 3$ that caused by old BHCV.

\section{References}

[1] J. Brod, M. Gorbahn and E. Stamou, Phys. Rev. D 83, 034030 (2011).

[2] J. Comfort et al., http://koto.kek.jp/pub/p14.pdf

[3] J. K. Ahn et al., Phys. Rev. D, 81, 072004 (2010).

[4] H. Fukui, et al., Nucl. Instr. and Methods in Phys. Research A, 419, 497-502 (1998). 